\title{
Differential Selective Etching of Functional Groups Strategy for Preparation of Organic Hollow Porous Silica
}

\author{
Jie Li ${ }^{1,}{ }^{*}$, Jia Zhang ${ }^{2}$, ZhuoQiong Wen ${ }^{1}$, Cui $\mathrm{Yu}^{1}$ and XiaoPeng $\mathrm{Li}^{1}$ \\ ${ }^{1}$ School of Urban Construction, Wuchang Institute of Technology, 430065 Wuhan, China \\ ${ }^{2}$ School of Food and Biological Engineering, Hubei University of Technology, 430068 Wuhan, China
}

\begin{abstract}
In this study, different structural of organic hollow porous silica were prepared based on differential selective etching of functional groups strategy, using ammonia as catalyst, cetyltrimethylammonium bromide $(\mathrm{CTAB})$ as pore-forming agent and emulsifier, cyano silica $\left(\mathrm{CN}-\mathrm{SiO}_{2}\right)$ or vinyl silica $\left(\mathrm{V}-\mathrm{SiO}_{2}\right)$ formed by hydrolysis condensation of 2-cyanoethyl triethoxysilane (CTES) or vinyl triethoxysilane (VTES) were used as cores, $\mathrm{NH}-\mathrm{SiO}_{2}$ prepared by 3 -aminopropyl triethoxysilane (APTES), $\mathrm{SH}-\mathrm{SiO}_{2}$ prepared by mercaptopropyl trimethoxysilane (MPTMS) and $\mathrm{UD}-\mathrm{SiO}_{2}$ prepared by urea-propyl triethoxysilane (UPTES) as shells, separately. The morphology and structure of the products were characterized by scanning electron microscopy(SEM) and transmission electron microscopy(TEM). The study results showed that $\mathrm{CN}-\mathrm{SiO}_{2}$ as core was considered to be benefit for the preparation of organic hollow porous silica compared to $\mathrm{V}-\mathrm{SiO}_{2}$ as core. In addition, core-shell structure obtained from $\mathrm{CN}$ $\mathrm{SiO}_{2} \mathrm{NH}-\mathrm{SiO}_{2}$ tend to be changed into hollow porous structure in alkaline condition on account of differential selective etching of functional groups strategy.
\end{abstract}

\section{Introduction}

Organic hollow porous silica nanospheres (OHPSNs) have increasingly attracted researchers' attention for their great potential application in many fields, such as drug delivery, catalysis, adsorbing materials and nanoreactors [1-5], due to their large capacity, low toxicity, excellent biocompatibility and easy modifification.

In general, for the process of fabricating hollow structure, the conventional strategies contain Kirkendall effect, hard/soft templating methods, etc. [6-10]. Compared with these methods, new emerging selftemplating method based on surface-protected etching or selective etching strategy has advantages of controlling morphology and simpler procedures. Yin and co-workers [11] have developed a surface-protecting etching strategy by using PVP as the protective agent. Shi's groups [12] have developed a structural difference-based strategy to fabricate HSNs. Recently, a cationic surfactant assisted selective etching strategy developed by Zheng [13] has also been proved to be simple and effective. However, to our knowledge, it's diffificult to prepare organic functionalized HSNs by these methods.

In addition, cetyltrimethylammonium bromide (CTAB) is a general cationic surfactant in the synthesis of hollow or porous silica since its diverse functions (structure-directing agent, pore-making agent and softtemplate and easy removal by calcination and extraction[14-15]. Especially, in the work of Zheng's group, CTAB not only played a important role in stabilizing the surface of HNSs from alkaline etching, but also realized the formation of worm-like mesopores shell[13]. It is believed that the potential value of CTAB in fabricating hollow structure remains to be further explored.

In this study, different kinds of OHPSNs with welldefined morphology were fabricated effffectively based on "functional group difference based selective etching strategy" using $\mathrm{CTAB}$ as pore-forming agent and emulsifier. In this method, bifunctional organic core/shell nanospheres were firstly prepared via a twostep sol-gel reaction in one pot. And then, the structural stability between core and sell was different because of the introduction of organic group, and the part whose alkali resistance was relatively weak would be dissolved preferentially, resulting in hollow porous silica with organic groups.

\section{Experiments}

\subsection{Chemicals and Reagents}

Cyanoethyltriethoxysilane (CTES, 95\%),

Vinyltriethoxysilane (VTES, 99\%), tetraethyl orthosilicate (TEOS, $98 \%$ ) Mercaptopropyltrimethoxysilane (MPTMS, 97\%), 3-Aminopropyltriethoxysilane (APTES) and Ureapropyl triethoxysilane were purchased from Aladdin.

*orresponding author: 544966911@qq.com 
Aqueous ammonia solution (28 wt $\%$ ), CTAB and $\mathrm{HCl}(37 \%)$ were obtained from Sinopharm Chemical Reagent

Company Ltd (China). All materials were of analytical grade and were used without further purifification. Deionized water was used in all experiments.

\subsection{Synthesis of $\mathrm{CN}-\mathrm{SiO}_{2} @ O$ Organo-silica or V- $\mathrm{SiO}_{2} @ O r g a n o-s i l i c a$ core-shell structures}

In a typical synthesis procedure, the desired amount of CTAB was dissolved in $50 \mathrm{~mL}$ of water at $30{ }^{\circ} \mathrm{C}$ under vigorous stirring, after the surfactant was fully dissolved, and uniform emulsion was formed. And then, $1 \mathrm{ml}$ of VTES or CTES was slowly added under vigorous stirring, after $1 \mathrm{~h}, 0.5 \mathrm{ml}$ of ammonia solution (28 wt\%) was added into the above solution slowly. The resultant mixture was stirred at $30^{\circ} \mathrm{C}$ for $6 \mathrm{~h}$. After a reaction time of about $6 \mathrm{~h}, 1 \mathrm{ml}$ of TEOS was added slowly into above mixtures, and $0.5 \mathrm{ml}$ of organosilane was successively injected immediately ten minutes later. After stirring at $30{ }^{\circ} \mathrm{C}$ for another $3 \mathrm{~h}$, white products were collected by centrifugation and washed with water and ethanol (95\%) and dried under $65^{\circ} \mathrm{C}$.

\subsection{Preparation of OHPSNs}

The solid products obtained above were collected and then acid extracted $(\mathrm{HCl})$ by reflfluxing in ethanol for 12 $\mathrm{h}$ to completely remove the surfactant. The materials were filtered, washed with water and then air dried at 50 ${ }^{\circ} \mathrm{C}$ to obtain the final surfactant-free products. The obtained particles without surfactant were further ultrasonically dispersed into sodium hydroxide aqueous $(0.05 \mathrm{M})$, and then stirred at $60{ }^{\circ} \mathrm{C}$ for $6 \mathrm{~h}$ to fabricate OHPSNs.

\subsection{Characterization methods}

Scanning electron microscopy images were determined by JSM-5610LV microscope. Transmission electron microscopy (TEM) was conducted with a JEM $2100 \mathrm{~F}$ electron microscope operated at $200 \mathrm{kV}$.

\section{Results and Discussions}

\subsection{The formation mechanism of OHPSNs}

In this study, a fundamental condition for the synthesis of OHPSNs is that an the structural different stability between core and sell, due to the introduction of organic group, and the part whose alkali resistance was relatively weak would be dissolved preferentially. their typical synthesis is schematically illustrated in Scheme 1. Firstly, hydrolysis and condensation reaction of CTES or VTES as core were performed in the presence of CTAB under alkaline conditions. After $6 \mathrm{~h}$, co-concentration reaction of TEOS and organosilane were proceeded, in order to prepare organic core-shell structures. Secondly, extraction experiment was adopted to remove surfactant $\mathrm{CTAB}$, resulting in the formation micropores on the interior and surface of core-shell structure.

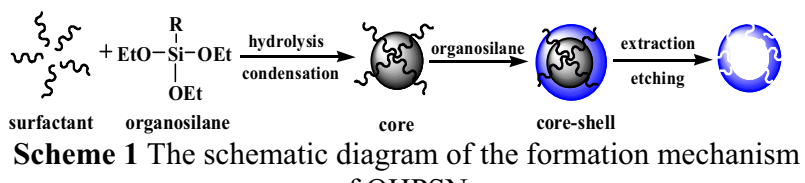
of OHPSNs

In the end, etching process was carried out under the condition that the strong basic was medium. The purpose of this process was to remove core material forming a type of nanostructures with hollow space inside, based on differential selective etching of functional groups strategy.

\subsection{Preparation and characterization of OHPSNs}

In this study, cyano silica $\left(\mathrm{CN}-\mathrm{SiO}_{2}\right)$ and vinyl silica $(\mathrm{V}$ $\mathrm{SiO}_{2}$ ) were used as cores, separately. The morphology and dispersity were different due to their differential hydrolysis and condensation in alkaline conditions.

\subsubsection{OHMSNs prepared with $\mathrm{CN}-\mathrm{SiO}_{2}$ as core}

The synthetic process for OHPSNs prepared with CN$\mathrm{SiO}_{2}$ as core was described as the above description. Herein, three shell materials were used, such as $\mathrm{NH}-\mathrm{SiO}_{2}$ prepared by APTES, $\mathrm{SH}-\mathrm{SiO}_{2}$ prepared by MPTMS, and $\mathrm{UD}-\mathrm{SiO}_{2}$ prepared by UPTES. The formed core-shell structures were further etched in strong basicity based on differential selective etching of functional groups strategy.

In our previous study, it was proved that $\mathrm{CN}-\mathrm{SiO}_{2}$ was fairly easy to hydrolyze in acidic or alkaline conditions[16]. Therefore, in order to obtain hollow porous silica, $\mathrm{CN}-\mathrm{SiO}_{2}$ was used as core in this study, other organosilanes were used as shell materials. The specific synthesis parameters for preparation OHPSNs using $\mathrm{CN}-\mathrm{SiO}_{2}$ as core were as follows.

Table 1 Specific synthesis parameters for preparation OHPSNs with different amounts of CTAB

\begin{tabular}{ccccc}
\hline $\begin{array}{c}\text { CTES } \\
(\mathrm{ml})\end{array}$ & $\begin{array}{c}\text { Organosilane } \\
(\mathrm{ml})\end{array}$ & $\begin{array}{c}\text { TEOS } \\
(\mathrm{ml})\end{array}$ & $\begin{array}{c}\mathrm{NH}_{3} \\
(\mathrm{ml})\end{array}$ & $\begin{array}{c}\text { CTAB } \\
(\mathrm{g})\end{array}$ \\
\hline 1 & 0.5 & 1 & 0.5 & 0.0168 \\
1 & 0.5 & 1 & 0.5 & 0 \\
\hline
\end{tabular}

a refers different organosilanes, such as APTES, VTES, MPTES and UPTES

Herein, the effect of the amounts of CTAB as poreforming agent and emulsifier on the formation of coreshell changed into porous structures has been studied. The amount of CTAB was shown in table1, in which $0.0168 \mathrm{~g}$ of $\mathrm{CTAB}$ in this system was the corresponding the critical micelle concentration (CMC) value. The fig. 1 showed the SEM images of of $\mathrm{CN}-\mathrm{SiO}_{2} @ \mathrm{NH}_{2}-\mathrm{SiO}_{2}$ core-shell structures with different magnification (A) low magnification and (A1) high magnification prepared using 0.0168g CTAB, and $\mathrm{CN}-\mathrm{SiO}_{2} @ \mathrm{NH}_{2}-$ $\mathrm{SiO}_{2}$ core-shell structures without CTAB. 

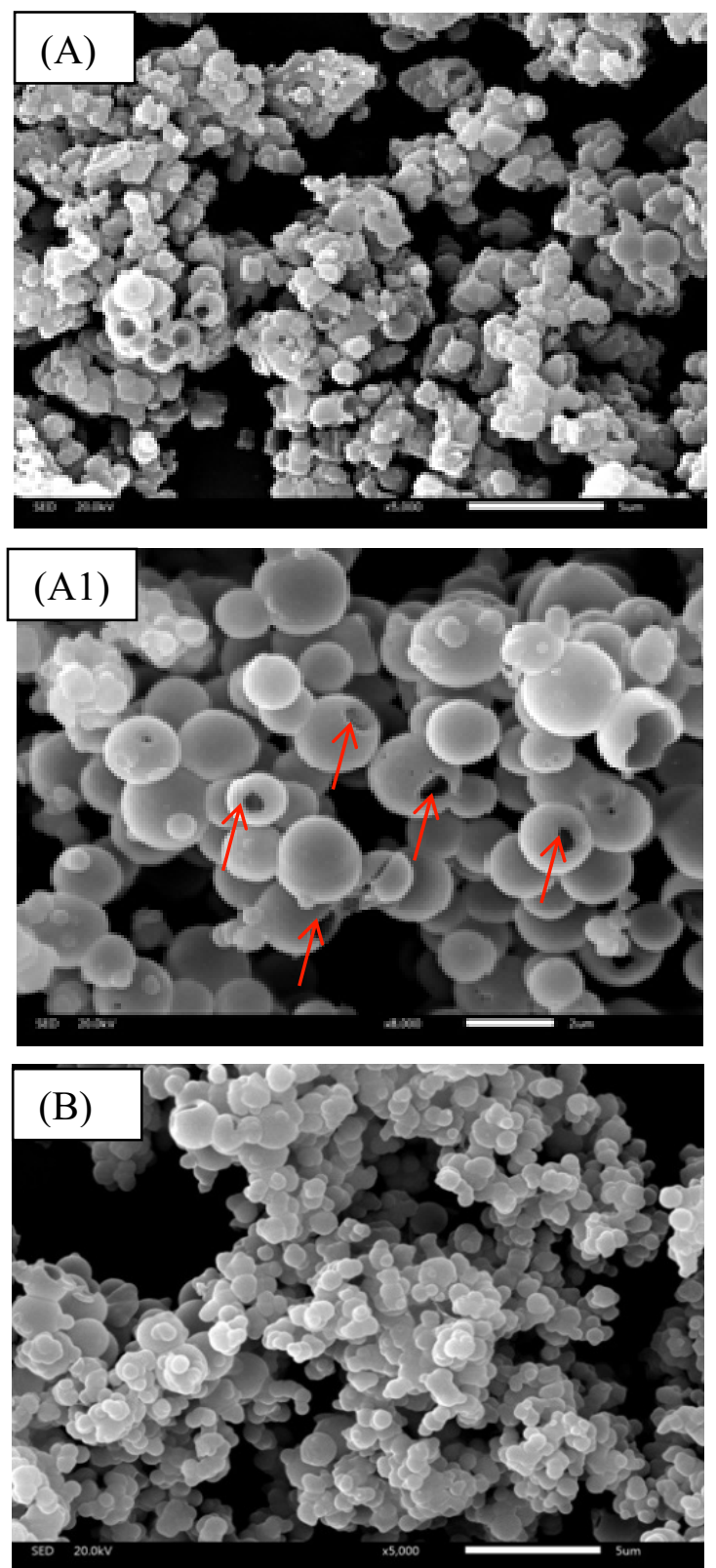

Fig. 1 The SEM images of CN-SiO $@ \mathrm{NH}_{2}-\mathrm{SiO}_{2}$ core-shell structures prepared $0.0168 \mathrm{~g}$ of CTAB with different magnification (A) low magnification and (A1) high magnification, and (B) $0 \mathrm{~g}$ of CTAB

More interestingly, in this study, it was found that $\mathrm{CN}-\mathrm{SiO}_{2} @ \mathrm{NH}_{2}-\mathrm{SiO}_{2}$ core-shell structure itselfwas hollow porous without etched when $0.0168 \mathrm{~g}$ of CTAB was used. To our knowledge, it was concerned with amino base group. It may be hydrolyzed and decomposed in the synthesis process of CN-SiO ${ }_{2} @ \mathrm{NH}_{2}-$ $\mathrm{SiO}_{2}$ core-shell structure. The specific morphology was shown in the fig.2 (A,A1). It was scanning electron microscope (SEM) images at different magnification in Fig.2 (A, A1). It was clearly observed that a void and broken shell structure on the surface of particles. However, solid spheres with poor dispersion were noticed under the condition without CTAB, shown in fig.1(B).

These phenomena might be due to the formation of micelle in the system. Uniform spherical micelle structure could be obtained under CMC of CTAB, just as the formation process mechanism of OHPSNs, and then uniform spherical micelle was then wrapped by organic silica prepared by organosilane to form homogeneous similar to core-shell structure, which to the benefit of organocore-organoshell. On the contrast, incomplete hydrolysis and condensation reaction might be occurred without $\mathrm{CTAB}$, resulting in agglomeration of particles, just as observed in fig.1(B).

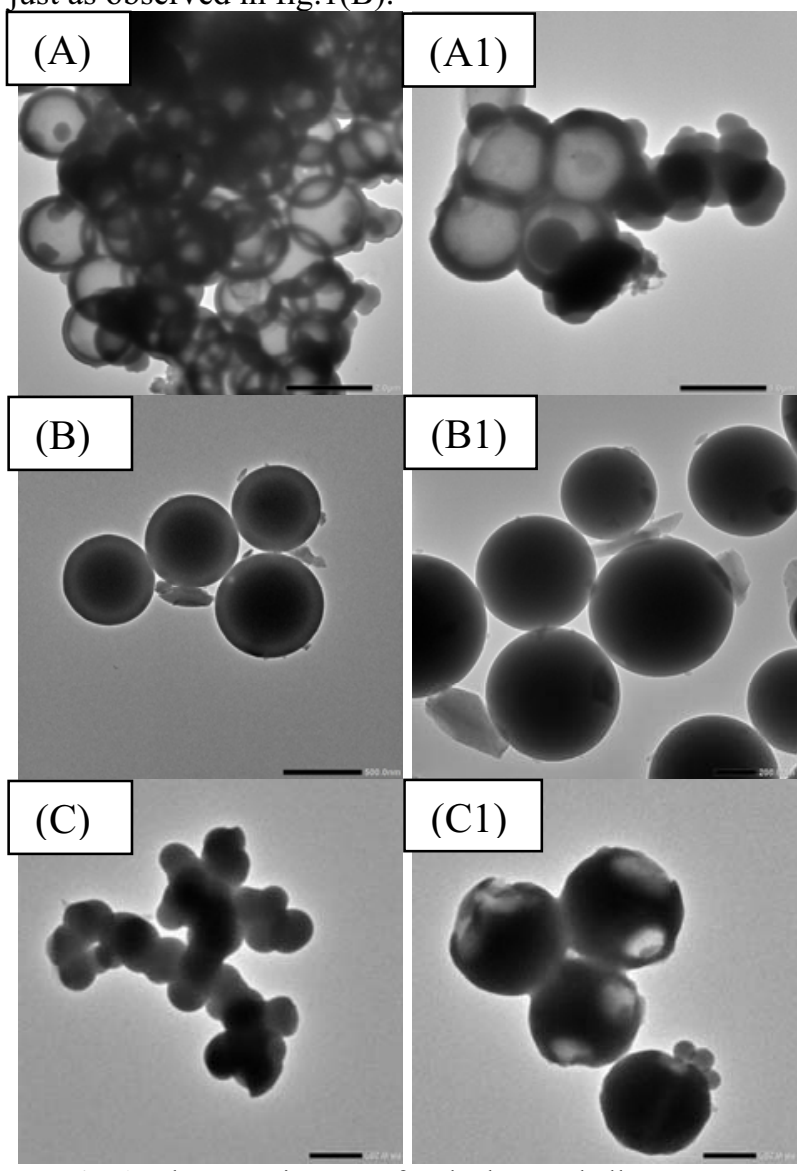

Fig. 2 The TEM images of etched core-shell structures

(A,A1) CN-SiO $@ \mathrm{NH}_{2}-\mathrm{SiO}_{2},(\mathrm{~B}, \mathrm{~B} 1) \mathrm{CN}-\mathrm{SiO}_{2} @ \mathrm{SH}-\mathrm{SiO}_{2}$ and (C,C1) CN-SiO ${ }_{2} @ \mathrm{UD}-\mathrm{SiO}_{2}$

It was showed from the above TEM images that hollow porous silica could be obtained successfully when $\mathrm{NH}_{2}-\mathrm{SiO}_{2}$ was used as shell. While $\mathrm{SH}-\mathrm{SiO}_{2}$ was as shell, core-shell structure were only able to prepare. On the contrast, cores and shell were all damaged as UD- $\mathrm{SiO}_{2}$ was used as shell. It was proved that $\mathrm{CN}-\mathrm{SiO}_{2}$ was not completely wrapped by $\mathrm{UD}-\mathrm{SiO}_{2}$, or thin shells were formed on the surface of $\mathrm{CN}-\mathrm{SiO}_{2}$. On the contrary, the relatively thick $\mathrm{SH}-\mathrm{SiO}_{2}$ shell was formed on the surface layer of $\mathrm{CN}-\mathrm{SiO}_{2}$, resulting in difficult to etch. It was thus core-shell morphology was still observed.

The difference with the above is $\mathrm{NH}_{2}-\mathrm{SiO}_{2}$ as shell. From the view of the TEM images obtained this study, the well-defined hollow porous structure could be obtained when $\mathrm{NH}_{2}-\mathrm{SiO}_{2}$ was used as shell, $\mathrm{CN}-\mathrm{SiO}_{2}$ was used as core. It may be due to the role of the amino base group, which accelerated cyanide hydrolysis and decomposition.

\subsubsection{OHPSNs prepared with $\mathrm{V}-\mathrm{SiO}_{2}$ as core}

The synthetic process for OHPSNs prepared with V$\mathrm{SiO}_{2}$ as core was similar to that with $\mathrm{CN}-\mathrm{SiO}_{2}$ as core. 
The vinyl groups was also instability in a strongly alkaline system, it was thus considered $\mathrm{V}-\mathrm{SiO}_{2}$ prepared by hydrolysis and condensation of vinyltriethoxysilane (VTES) as core to synthesize OHPSNs based on the functional group differencebased selective etching strategy.

Fig.3 showed the SEM images of etched core-shell structures prepared with $\mathrm{V}-\mathrm{SiO}_{2}$ as core. And it was completely different from $\mathrm{CN}-\mathrm{SiO}_{2}$ as core to obtain products. Just as Fig. 3(a,a1), it was the SEM images of etched $\mathrm{V}-\mathrm{SiO}_{2} @ \mathrm{NH}_{2}-\mathrm{SiO}_{2}$, compared with that CN$\mathrm{SiO}_{2} @ \mathrm{NH}_{2}-\mathrm{SiO}_{2}$, showed from Fig. 3(a,a1) that the poor dispersion and serious aggregation, the uniform hollow porous structure was not found out.
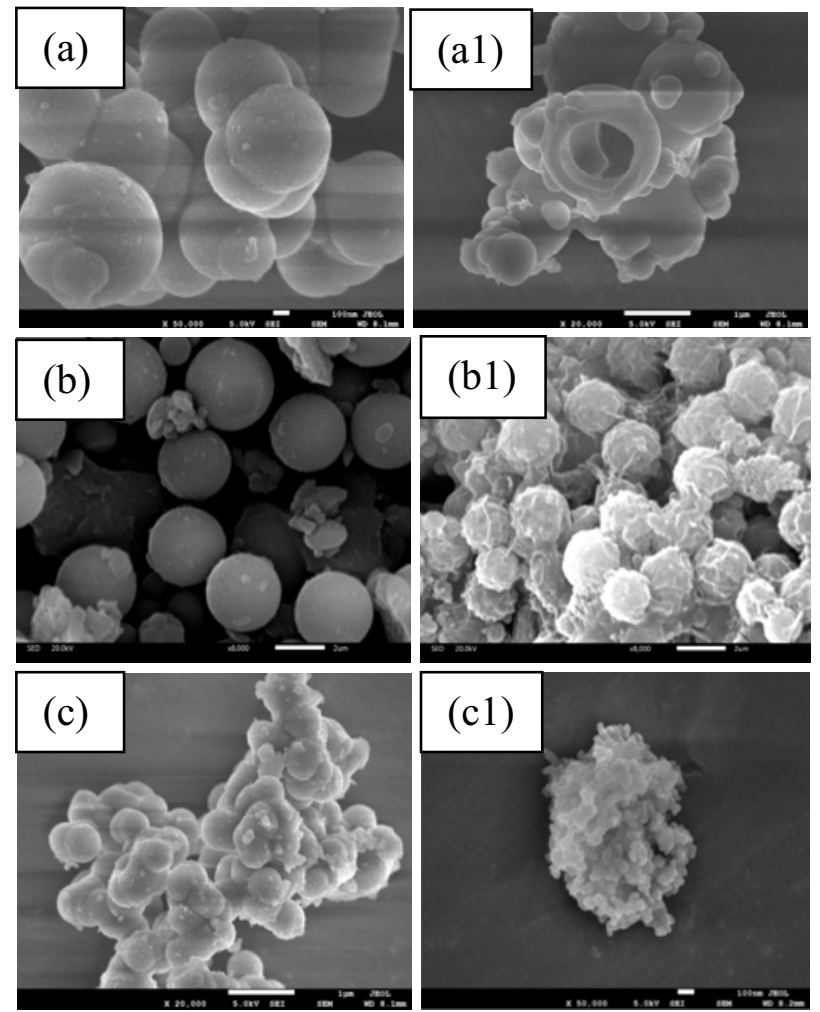

Fig. 3 The SEM images of etched core-shell structures (a,a1)V-SiO $\mathrm{S}_{2} @ \mathrm{NH}_{2}-\mathrm{SiO}_{2},(\mathrm{~b}, \mathrm{~b} 1) \mathrm{V}-\mathrm{SiO}_{2} @ \mathrm{SH}-\mathrm{SiO}_{2}$ and (c,c1) V-SiO $2 @$ UD-SiO 2

In addition, the SEM images of etched V-SiO $\mathrm{SiO}_{2}$ was shown in Fig.3(b,b1). Similarly, the particles with the well-defined morphology and great dispersibility were also observed when $\mathrm{V}-\mathrm{SiO}_{2}$ as core and $\mathrm{SH}-\mathrm{SiO}_{2}$ as shell. However, distinct pore structure and hollow structure were also not appeared in this product. What was worse, agglomeration state was only found while $\mathrm{V}-\mathrm{SiO}_{2}$ as core and $\mathrm{UD}-\mathrm{SiO}_{2}$ as shell, just observed in Fig. 3(c,c1). This was similar to what's happening in the structure of etched V-SiO $@ \mathrm{NH}_{2}-\mathrm{SiO}_{2}$.

\section{Conclusions}

In a word, it could be clearly found from the above research results that organic hollow porous silica nanospheres (OHPSNs) prepared by functional group differencebased selective etching strategy was relatively available when using $\mathrm{CN}-\mathrm{SiO}_{2}$ as core. This might be explained by the fact that $\mathrm{CN}-\mathrm{SiO}_{2}$ was more unstable in alkaline conditions compared with $\mathrm{V}-\mathrm{SiO}_{2}$ as core.

\section{Acknowledgements}

We are grateful for the financial support of the Nature Science Foundation of Hubei Province (No. 2017CFB296) and Outstanding Young and Middle-aged Scientific and Technological Innovation Team in Universities of Hubei Province (No.T201939). This work was also financially supported by the Guangdong Well-SilicaSol Co., LTD, China.

\section{References}

1. J.S. Gao, X.Y. Zhang, Y. Lu, S.M. Liu, J. Liu, Chem Eur J, 21 (2015)

2. A.M. El-Toni, M.A. Habila, M.A. Ibrahim, J.P. Labis, Chem Eng J, 251(2014)

3. S. Shi, M. Wang, C. Chen, F. Lu, X. Zheng, J. Gao, RSC Adv, 3(2013)

4. M. Sasidharan, H. Zenibana, M. Nandi, A. Bhaumik, K. Nakashima, Dalton T, 42(2013)

5. Z.G. Teng, X.D. Su, Y.Y. Zheng, J.J. Zhang, Y. Liu, S.J. Wang, J. Wu, G.T. Chen, J.D. Wang, D.Y. Zhao. J Am Chem Soc, 137 (2015)

6. J. Yu, W. Liu, H. Yu, Cryst. Growth Des. 8 (2008)

7. T.S. Deng, F. Marlow, Chem Mater, 24 (2012)

8. G. Qi, Y. Wang, L. Estevez, A.K. Switzer, X. Duan, X. Yang, E.P. Giannelis, Chem Mater, 22 (2010)

9. Y. Yamada, M. Mizutani, T. Nakamura, K. Yano, Chem Mater, 22 (2010)

10. J. Liu, S.B. Hartono, Y.G. Jin, Z. Li, G.Q. Lu, S.Z. Qiao, J Mater Chem, 20 (2010)

11. Q. Zhang, J.-P. Ge, J. Goebl, Y.-X. Hu, Z.-D. Lu, Y.-D. Yin, Nano Res. 2 (2009)

12. Y. Chen, H.-R. Chen, L.-M. Guo, Q.-J. He, F. Chen, J. Zhou, J.-W. Feng, J.-L. Shi, Acs Nano, 4 (2010)

13. X.-L. Fang, C. Chen, Z.-H. Liu, P.-X. Liu, N.-F. Zheng, Nanoscale, 3 (2011)

14. Y. Wang, P.P. Wang, L.X.Chen, J. Li, Z.H. Liu, J Solid State Chem, 266 (2018)

15. Z.G. Teng, X.D. Su, Y.Y. Zheng, J.J. Zhang, Y. Liu, S.J. Wang, J. Wu, G.T. Chen, J.D.Wang, D.Y. Zhao, G.M. Lu, J Am Chem Soc, 137 (2015)

16. Y. Wang, Z.H. Liu, L.X.Chen, J. Li, P.P. Wang, J Sol-Gel Sci Tech, 87(2018) 